\title{
О СВЯЗЯХ МЕЖДУ ФУНКЦИОНАЛЬНЫМИ ИСЧИСЛЕНИЯМИ БОХНЕРА - ФИЛЛИПСА И ХИЛЛЕ - ФИЛЛИПСА
}

\section{А. Р. Миротин}

\begin{abstract}
Классическое функциональное исчисление Хилле - Филлипса генераторов $C_{0}$-полугрупп, изложенное в известной монографии этих авторов, имеет в качестве символов преобразования Лапласа мер и приводит к ограниченным операторам. Другое важное функциональноте исчисление генераторов полугрупп исчисление Бохнера - Филлипса - использует в качестве символов функции Бернштейна. В работе дается расширение функционального исчисления Хилле-Филлипса, приводящее к неограниченным операторам. Указаны связи этого исчисления с исчислением Бохнера-Филлипса. В частности, для случая генераторов равномерно устойчивых полугрупп доказаны правило произведения и теорема о композиции понимаемых в разных смыслах функций. Получены условия обратимости операторов, возникающих в исчислении Бохнера - Филлипса. Рассмотрены примеры. Сформулированы нерешенные задачи.

Ключевые слова: функциональное исчисление Хилле-Филлипса, функциональное исчисление Бохнера-Филлипса, дробные степени операторов, подчиненная полугруппа.
\end{abstract}

A. R. Mirotin. On connections between the Bochner-Phillips and Hille-Phillips functional calculi.

The classical Hille-Phillips functional calculus of generators of $C_{0}$-semigroups, presented in the well-known monograph of these authors, uses Laplace transforms of measures as symbols and leads to bounded operators. Another important functional calculus of semigroup generators - the Bochner-Phillips calculus - uses Bernstein functions as symbols. In this work, an extension of the Hille-Phillips functional calculus is considered that leads to unbounded operators. Connections of this calculus with the Bochner-Phillips functional calculus are indicated. In particular, for generators of uniformly stable semigroups, the multiplication rule and the composition theorem are proved for functions understood in different senses. Conditions for the invertibility of operators that arise in the Bochner-Phillips calculus are obtained. Several examples are given and unsolved problems are formulated.

Keywords: Hille-Phillips functional calculus, Bochner-Phillips functional calculus, fractional powers of operators, subordinate semigroup.

MSC: 47A60, 47D03

DOI: $10.21538 / 0134-4889-2020-26-3-118-132$

\section{Введение}

Известное функциональное исчисление Хилле-Филлипса генераторов однопараметрических полугрупп операторов $[1$, гл. 15,16$]$ использует в качестве символов класс функций, представимых в виде преобразований Лапласа

$$
L a(s):=\int_{0}^{\infty} e^{s t} d a(t) \quad\left(s<\omega_{0}\right),
$$

$\sigma$-конечных комплексных регулярных борелевских мер $a$ на $\mathbb{R}$, сосредоточенных на $\mathbb{R}_{+}$. (Пространство таких мер далее будет обозначаться через $\mathcal{M}\left(\mathbb{R}_{+}\right)$.) При этом условия, налагаемые в [1] на меру и полугруппу, приводят к тому, что возникающие в результате операторы ограничены. Там же (с. 463) поставлена задача построения такого расширения этого исчисления, которое бы приводило к неограниченным операторам. Это вызвало поток работ в данном направлении (см, например, [2-7] и списки литературы в указанных работах). 
Другое важное функциональное исчисление генераторов полугрупп, исчисление Бохнера Филлипса, использует в качестве символов функции Бернштейна (см., например, [8;9], а также монографию [10, гл. 13], где рассмотрен случай сжимающих полугрупп). В данной работе рассматривается версия, использующая отрицательные функции Бернштейна [9]. Связи этого исчисления с голоморфным исчислением и исчислением Хирша рассматривались, например, в [11].

Основной целью статьи является установление связей между исчислениями БохнераФиллипса и Хилле-Филлипса, в частности доказательство правила умножения и теоремы о сложной функции. В качестве следствия получается правило обращения операторов, возникающих в исчислении Бохнера - Филлипса. Для этих целей оказалось удобным класс символов, используемых в исчислении Хилле-Филлипса, расширить так, что возникающие операторы могут быть и неограниченными. Аналогичная задача для генераторов групп рассматривалась в [2]. Подход, основанный на другом классе символов (причем для наборов нескольких коммутирующих генераторов), представлен в [12].

Всюду ниже $A$ есть генератор ограниченной $C_{0}$-полугруппы $T$ в банаховом пространстве $X$ с областью определения $D(A)$ и образом $\operatorname{Im}(A)$. Существенной областью оператора $A$ мы называем такое линейное многообразие $L \subset D(A)$, что $A$ есть замыкание своего сужения на $L$. Через $L B(Y, X)$ обозначается пространство линейных ограниченных операторов, действующих между банаховыми пространствами $Y$ и $X, L B(X):=L B(X, X)$. Если $f-$ функция на $\mathbb{R}_{+}$, то через $\widehat{f}$ будет обозначаться преобразование Лапласа меры $f(t) d t$. Для регулярной положительной борелевской меры $\mu$ на $\mathbb{R}_{+}$через $\mu(t)$ обозначается ее функция распределения, непрерывная слева (т. е. $\mu(t)=\mu([0, t))$ при $t>0)$. При этом нам будет удобно считать, что $\mu(t)=0$ при $t \leq 0$. Через $L \mathcal{M}$ будем обозначать класс функций, для которых представление $(0.1)$ справедливо при $\omega_{0}=0$. Результаты работы частично анонсированы в [13].

\section{1. Расширение исчисления Хилле - Филлипса}

Данный раздел носит вспомогательный характер. В нем мы вводим расширенное исчисление Хилле-Филлипса и даем условия замкнутости возникающих операторов. Нижеследующее определение формально совпадает с определением, предложенным Хилле и Филлипсом в монографии [1], но мы отказываемся от наложенных там ограничений, гарантирующих существование интеграла и ограниченность определяемого им оператора (см. также работу [2], посвященную генераторам групп).

О п р е д е л е н и е 1 . Для функции $g$ из $L \mathcal{M}, g=L a\left(a \in \mathcal{M}\left(\mathbb{R}_{+}\right)\right)$положим

$$
g(A) x=\int_{0}^{\infty} T(t) x d a(t),
$$

где область определения $D_{0}(g(A))$ этого оператора состоит из тех $x \in X$, для которых интеграл в правой части существует в смысле Бохнера.

Следующий пример иллюстрирует определение 1.

П р и м е р 1. Пусть $g(s)=s^{-1}, s<0$. Тогда $g=L a$, где $d a(t)=-d t(d t-$ мера Лебега на $\left.\mathbb{R}_{+}\right)$. Таким образом, в силу определения 1 при $x \in D_{0}(g(A))$

$$
g(A) x=-\int_{0}^{\infty} T(t) x d t .
$$

Предположим, что генератор $A$ инъективен, а полугруппа $T$ сильно устойчива (т. е. $T(t) y \rightarrow 0$ при $y \in X, t \rightarrow \infty)$, и покажем, что из определения 1 следует равенство

$$
g(A)=A^{-1} .
$$


Пусть $x \in \operatorname{Im}(A)$ и $y=A^{-1} x$. Тогда

$$
g(A) x=-\int_{0}^{\infty} T(t) A y d t=-\int_{0}^{\infty} d T(t) y=y .
$$

Таким образом, $D_{0}(g(A)) \supseteq \operatorname{Im}(A)$ и при $x \in \operatorname{Im}(A)$ имеем $g(A) x=A^{-1} x$. Нам осталось доказать включение $D_{0}(g(A)) \subseteq \operatorname{Im}(A)$. С этой целью выберем произвольно $x \in D_{0}(g(A))$ и рассмотрим последовательность

$$
y_{n}:=-\int_{0}^{n} T(t) x d t
$$

Положим $y:=\lim _{n \rightarrow \infty} y_{n}$. Как известно, $y_{n} \in D(A)$ и

$$
A y_{n}=-A \int_{0}^{n} T(t) x d t=x-T(n) x .
$$

Поэтому $A y_{n} \rightarrow x(n \rightarrow \infty)$, и в силу замкнутости оператора $A$ имеем $y \in D(A)$ и $A y=x$, что и завершает доказательство.

Теорема 1. Пусть $g=L a \in L \mathcal{M}$. Тогда справедливы следующие утверждения.

1) Eсли $\operatorname{Im}(A) \subset D_{0}(g(A))$, то оператор $g(A) A$ ограничен относительно $A$.

2) Если дополнительно предположить, что оператор $A$ ингективен, то $g(A)$ замкнут на подпространстве $D(A) \cap D_{0}(g(A))$, наделенном нормой графика.

3) Если $\int_{0}^{\infty}\|T(t)\| d|a|(t)<\infty$, то оператор $g(A)$ ограничен на $X$ и рассматриваемое исчисление согласовано с классическим исчислением Хилле-Филлипса. В частности, это имеет место, если полугруппа $T$ равномерно устойчива $($ m. е. $\|T(t)\| \rightarrow 0$, при $t \rightarrow \infty)$.

Д о к а з а т е л ь с т в о. 1) Для любого $x \in D(A)$ определим операторы

$$
B_{n} x=\int_{0}^{n} T(t) A x d a(t)
$$

(интеграл понимается в смысле Бохнера). Поскольку у нас $A x \in D_{0}(g(A))$, то $B_{n} x \rightarrow g(A) A x$ $(n \rightarrow \infty)$ при всех $x \in D(A)$. Далее, так как оператор $A$ замкнут, то пространство $Y=D(A)$, наделенное нормой графика $\|x\|_{Y}=\|x\|+\|A x\|$, банахово. Кроме того, $B_{n} \in L B(Y, X)$ ввиду

$$
\left\|B_{n} x\right\| \leq\left(\int_{0}^{n}\|T(t)\| d|a|(t)\right)\|x\|_{Y} .
$$

В силу теоремы Банаха-Штейнгауза оператор $g(A) A$ тоже принадлежит $L B(Y, X)$, т. е. для некоторой константы $C>0$ выполняется неравенство ограниченности $\|g(A) A x\| \leq C\|x\|_{Y}$ $(x \in Y)$, это и означает, что оператор $g(A) A$ ограничен относительно $A$ (см., например, [14, c. 241]).

2) Заметим сначала, что при $x \in D(A) \cap D_{0}(g(A))$ справедливо равенство

$$
A g(A) x=g(A) A x .
$$

Действительно, с учетом замкнутости $A$ и сходимости интегралов имеем

$$
g(A) A x=\int_{0}^{\infty} T(t) A x d a(t)=\int_{0}^{\infty} A T(t) x d a(r)=A g(A) x,
$$


поскольку $\operatorname{Im}(A) \subset D_{0}(g(A))$. Теперь если $A$ инъективен, то с помощью утверждения 1$)$ получаем, что оператор $g(A) x=A^{-1} g(A) A x$ замкнут на подпространстве $D(A) \cap D_{0}(g(A))$ пространства $Y$ как произведение замкнутого и ограниченного операторов.

3) Первое утверждение следует из свойств интеграла Бохнера. Если же полугруппа $T$ равномерно устойчива, то справедлива оценка $\|T(t)\| \leq M e^{-\sigma t}$, где $\sigma>0$. Поэтому интеграл в определении $g(A) x$ существует в смысле Бохнера при всех $x \in X$ и оператор $g(A)$ ограничен, так как

$$
\|g(A) x\| \leq \int_{0}^{\infty}\|T(t) x\| d|a|(t) \leq M\|x\| \int_{0}^{\infty} e^{-\sigma t} d|a|(t)
$$

(последний интеграл сходится, поскольку по условию теоремы $L a \in L \mathcal{M}$; это по определению означает, что интеграл в (0.1) существует при всех $s<0)$.

Теорема доказана.

Всюду далее для функции $f$ на $\mathbb{R}_{+}$через $\widehat{f}$ обозначается ее преобразование Лапласа, т. е.

$$
\widehat{f}(s):=\int_{0}^{\infty} e^{s t} f(t) d t \quad(s<0) .
$$

Следствие 1. Пусть $g=\widehat{f}$ есть преобразование Лапласа функции $f$, причем при $x$ из $D(A)$ существуют $\lim _{n \rightarrow \infty} f(n) T(n) x u \int_{0}^{\infty} T(t) x d f(t)$. Тогда $\operatorname{Im}(A) \subset D_{0}(g(A))$ и справедливы все утверждения теоремы 1.

Д о к а з а т е л ь с т в о. Интегрируя по частям, получаем при всех $x \in D(A)$

$$
\int_{0}^{n} T(t) A x f(t) d t=\int_{0}^{n} f(t) d T(t) x=f(n) T(n) x-f(0) x-\int_{0}^{n} T(t) x d f(t),
$$

причем правая часть имеет предел при $n \rightarrow \infty$.

Следствие доказано.

Следствие 2. Если $\operatorname{Im}(A) \subset D_{0}(g(A))$, то оператор $g(A) A$ ограничен, если ограничен оператор A.

Следствие 3. Если $\operatorname{Im}(A) \subset D_{0}(g(A))$ и оператор $A$ инбективен, то $g(A) \mid \operatorname{Im}(A)$ ограничен относительно $A^{-1}$.

Следствие 4. Если $\operatorname{Im}(A) \subset D_{0}(g(A))$ и существует ограниченный обратный оператор $A^{-1}$ на $\operatorname{Im}(A)$, то $g(A)$ ограничен на $\operatorname{Im}(A)$.

Теорема 2. Пусть $g=\widehat{f}$, где функиия $f$ такова, что оператор

$$
C y:=\int_{0}^{\infty} T(t) y d f(t)
$$

ограничен на $\operatorname{Im}(A)$, а последовательность $f(n) T(n)$ сходится на $D(A)$ поточечно к операmору $B \in L B(D(A))$. Тогда

1) $\operatorname{Im}(A) \subset D_{0}(g(A))$ и оператор $g(A) A$ ограничен;

2) если оператор $A$ ингективен, то оператор $g(A) \mid D(A) \cap D_{0}(g(A))$ замкнут, а если еще $B=0$, то замкнут также и оператор $g(A) \mid \operatorname{Im}(A)$. 
Д о к а з а т е л ь с т в о. 1) Включение $\operatorname{Im}(A) \subset D_{0}(g(A))$ сразу вытекает из следствия 1. Переходя к пределу в формуле (1.1), получаем при $x \in D(A)$

$$
g(A) A x=\int_{0}^{\infty} T(t) A x f(t) d t=B x-f(0) x-\int_{0}^{\infty} T(t) x d f(t),
$$

откуда и следует ограниченность $g(A) A$ на $D(A)$.

2) Здесь первое утверждение выводим из п. 1) и равенства $g(A) x=A^{-1} g(A) A x(x \in D(A) \cap$ $\left.D_{0}(g(A))\right)$, как в доказательстве теоремы 1 . Пусть теперь $B=0$. Полагая в (1.2) $y=A x$, имеем

$$
g(A) y=-f(0) A^{-1} y-\int_{0}^{\infty} T(t) A^{-1} y d f(t) .
$$

Но оператор

$$
y \mapsto A \int_{0}^{\infty} T(t) A^{-1} y d f(t)=\int_{0}^{\infty} A T(t) A^{-1} y d f(t)=\int_{0}^{\infty} T(t) y d f(t)=C y
$$

ограничен на $\operatorname{Im}(A)$, а потому оператор

$$
y \mapsto g(A) y=-A^{-1}(f(0) y+C y)
$$

замкнут на $\operatorname{Im}(A)$ как произведение замкнутого и ограниченного операторов.

Теорема доказана.

П р и м е ч а н и е. См. также следствие 5 ниже.

\section{2. Связь с исчислением Бохнера - Филлипса}

Следующие теоремы устанавливают связь между рассматриваемым исчислением и исчислением Бохнера - Филлипса генераторов полугрупп, символами в котором являются функции Бернштейна (см. определение 2 , а также, например, [9]). Ниже через $\mathcal{T}$ будет обозначаться класс отрицательных функций Бернштейна одного переменного. Функция $\psi$ из $\mathcal{T}$ определена и отрицательна на $(-\infty, 0)$, аналитически продолжается в левую полуплоскость комплексной плоскости и допускает там интегральное представление

$$
\psi(z)=c_{0}+\int_{0}^{\infty}\left(e^{z u}-1\right) u^{-1} d \rho(u) \quad(\operatorname{Re} z<0),
$$

где $c_{0}=\psi(-0) ; \rho-$ положительная мера на $\mathbb{R}_{+}$, причем $\int_{0}^{r} d \rho(u)<\infty, \int_{r}^{\infty} u^{-1} d \rho(u)<\infty$ при $r>0$.

Например, функции $-(-s)^{\beta}(0 \leq \beta \leq 1),-\log (1-s)$ и $-i \arccos (1-s)$ принадлежат $\mathcal{T}$ (см. $[9 ; 15])$.

Отметим, что $\psi \in \mathcal{T}$, если и только если функция $t \mapsto-\psi(-t)$ является (положительной) функцией Бернштейна в том смысле, который придается этому понятию, например, в монографии [10].

О п р е д е л е н и е 2. Для отрицательной функции Бернштейна $\psi$ с интегральным представлением (2.1) и генератора $A$ ограниченной $C_{0}$-полугруппы $T$ на банаховом пространстве $X$ ее значение на операторе $A$ при $x \in D(A)$ определяется интегралом Бохнера

$$
\psi(A) x=c_{0} x+\int_{\mathbb{R}_{+}}(T(u)-I) x u^{-1} d \rho(u) .
$$


При этом замыкание этого оператора, также обозначаемое через $\psi(A)$, существует и является генератором ограниченной $C_{0}$-полугруппы $g_{t}(A)$ на $X$. Здесь $g_{t}(s):=e^{t \psi(s)}$, а оператор $g_{t}(A)$ понимается в смысле исчисления Хилле-Филлипса, поскольку функция $g_{t}(s)$ абсолютно монотонна на $(-\infty, 0]$ (последнее означает, что все ее производные неотрицательны, см., например, [16, гл. 4]), а потому по классической теореме Бернштейна - Уиддера есть преобразование Лапласа некоторой субвероятностной меры $\nu_{t}$ (т. е. положительной меры, для которой $\left.\nu_{t}\left(\mathbb{R}_{+}\right) \leq 1\right)^{1}$.

Теорема 3. Пусть $\psi \in \mathcal{T}, \psi(0)=0$. Тогда функиия $\tilde{\psi}(s):=\psi(s) / s$ принадлежит $L \mathcal{M} u$ при всех $x \in D(A) \cap D_{0}(\tilde{\psi}(A))$ справедливо равенство

$$
\psi(A) x=A \tilde{\psi}(A) x=\tilde{\psi}(A) A x
$$

где $\tilde{\psi}(A)$ понимается в смысле определения 1 , а $\psi(A)$ - в смысле исчисления БохнераФиллипса.

Д о к а з а т е л ь с т в о. В силу формулы (2.1) и теоремы Тонелли

$$
\begin{gathered}
\tilde{\psi}(s)=\int_{0}^{\infty} \frac{e^{s u}-1}{s} u^{-1} d \rho(u) \\
=\int_{0}^{\infty}\left(\int_{0}^{\infty} 1_{[0 ; u]}(r) e^{s r} d r\right) u^{-1} d \rho(u)=\int_{0}^{\infty} e^{s r}\left(\int_{0}^{\infty} 1_{[0 ; u]}(r) u^{-1} d \rho(u)\right) d r=\widehat{f}(s),
\end{gathered}
$$

где $f(r)=\int_{r}^{\infty} u^{-1} d \rho(u)$, а $1_{A}-$ индикатор множества $A$.

Следовательно, если $x \in D_{0}(\tilde{\psi}(A))$, то

$$
\tilde{\psi}(A) x=\int_{0}^{\infty} T(t) x f(t) d t .
$$

С другой стороны, при $x \in D(A) \cap D_{0}(\tilde{\psi}(A))$ по теореме Фубини для интеграла Бохнера

$$
\begin{gathered}
\psi(A) x:=\int_{0}^{\infty}(T(u)-I) x u^{-1} d \rho(u) \\
=\int_{0}^{\infty}\left(\int_{0}^{u} A T(t) x d t\right) u^{-1} d \rho(u)=\int_{0}^{\infty}\left(\int_{0}^{\infty} 1_{[0 ; u]}(t) A T(t) x d t\right) u^{-1} d \rho(u) \\
=A \int_{0}^{\infty} T(t) x\left(\int_{0}^{\infty} 1_{[0 ; u]}(t) u^{-1} d \rho(u)\right) d t=A \tilde{\psi}(A) x
\end{gathered}
$$

(теорема Фубини применима, так как последний повторный интеграл совпадает с интегралом Бохнера в (2.3), а потому абсолютно сходится).

Поскольку операторы $A$ и $T(t)$ перестановочны, аналогично доказывается и второе равенство.

Теорема доказана.

\footnotetext{
${ }^{1}$ Полугруппа $g_{t}(A)$ называется подчиненной полугруппе $T$, терминология мотивирована приложениями к теории вероятностей (см., например, [10]).
} 
Следствие 5. Если оператор А имеет ограниченный обратный, то сужение оператора $\tilde{\psi}(A)$ на подпространство $D(A) \cap D_{0}(\tilde{\psi}(A))$ есть замыкаемый оператор.

Д о к а з а т е л ь с т в о. Положим для краткости $D=D(A) \cap D_{0}(\tilde{\psi}(A))$. Первая из формул $(2.2)$ влечет равенство $\tilde{\psi}(A) x=A^{-1} \psi(A) x$ при $x \in D$. В силу теоремы 4.1 из [15] и ее следствия операторы $\psi(A)$ и $A^{-1}$ коммутируют. В частности, так как $D(A) \subset D(\psi(A))$, то при всех $x \in D(A) \supset D$ имеем $A^{-1} \psi(A) x=\psi(A) A^{-1} x$. Следовательно, справедливо равенство $\tilde{\psi}(A)\left|D=\psi(A) A^{-1}\right| D$ (вертикальная черта обозначает сужение), причем оператор $\psi(A) A^{-1}$ на своей максимальной области определения замкнут как произведение замкнутого и ограниченного операторов. Значит, оператор $\tilde{\psi}(A) \mid D$ имеет замкнутое расширение, и, стало быть, допускает замыкание (см., например, [14, с. 210]).

Следствие доказано.

Следствие 6. Onератор $\tilde{\psi}(A)$ отображает $D(A) \cap D_{0}(\tilde{\psi}(A))$ в $D(A)$.

Следствие 7. Oператор A отображает $D(A) \cap D_{0}(\tilde{\psi}(A))$ в $D_{0}(\tilde{\psi}(A))$.

Следствие 8. Если полугруппа $T$ равномерно устойчива, то оператор $\tilde{\psi}(A)$ ограничен и отображает $X$ в $D(A)$.

Д о к а з а т е ль с т в о. По теореме $1 D_{0}(\tilde{\psi}(A))=X$, оператор $\tilde{\psi}(A)$ ограничен и в силу $(2.2) \psi(A) x=A \tilde{\psi}(A) x$ при всех $x \in D(A)$. Поскольку операторы $\psi(A)$ и $A \tilde{\psi}(A)$, фигурирующие в обеих частях этого равенства, замкнуты, а подпространство $D(A)$ всюду плотно, это равенство выполняется при всех $x \in X$. Следовательно, $\tilde{\psi}(A) x \in D(A)$ при всех $x \in X$.

Следствие доказано.

Теорема 4. Пусть $g=L a \in L \mathcal{M}$, где $a-$ положительная $\sigma$-конечная борелевскал мера, а полугруппа $T$ равномерно устойчива. Если функиия $\psi \in \mathcal{T}$ имеет интегральное представление (2.1), причем функиия

$$
b(t):=\int_{0}^{\infty}(a(t-u)-a(t)) u^{-1} d \rho(u)
$$

имеет ограниченную вариачию на любом интервале вида $(0, R)$, mо $h:=g \psi \in L \mathcal{M}$, и при $x \in D(\psi(A))$ справедливы равенства $h(A) x=\psi(A) g(A) x=g(A) \psi(A) x$.

Д о к а з а т е л ь с т в о. Поскольку полугруппа $T$ равномерно устойчива, то найдутся такие постоянные $M>0, \sigma>0$, что $\|T(t)\| \leq M e^{-\sigma t}$ при всех $t \in \mathbb{R}_{+}$. А так как $L a(s)$ существует при всех $s<0$, то (см. [16, гл. 2, теорема 2.2a]) $a(t)=o\left(e^{c t}\right)$ при всех $c>0$. Поэтому в силу теоремы 1 оператор $g(A)$ ограничен. Заменяя при необходимости $\psi(s)$ на $\psi(s)-\psi(0)$, можно считать, что в формуле $(2.1) c_{0}=0$. Тогда $h=L b \in L \mathcal{M}$. В самом деле, так как по нашему соглашению для функции распределения $a(t)$ меры $a$ справедливо равенство $a(t)=0$ при $t \in(-\infty, 0]$, то, интегрируя по частям (см. [16, гл. 2, теорема 2.3a]), получаем при $s<0$

$$
g(s)=\int_{0}^{\infty} e^{s t} d a(t)=(-s) \int_{0}^{\infty} e^{s t} a(t) d t .
$$

Следовательно, при $u \geq 0$ и $s<0$ мы имеем

$$
\left(e^{s u}-1\right) g(s)=(-s) \int_{0}^{\infty}\left(e^{s u}-1\right) e^{s t} a(t) d t
$$




$$
=(-s)\left(\int_{0}^{\infty} e^{s(t+u)} a(t) d t-\int_{0}^{\infty} e^{s t} a(t) d t\right)=(-s) \int_{0}^{\infty} e^{s t}(a(t-u)-a(t)) d t .
$$

Поэтому по теореме Тонелли при $s<0(b(0)=0)$ выводим

$$
\begin{aligned}
& h(s)=\int_{0}^{\infty}\left(e^{s u}-1\right) g(s) u^{-1} d \rho(u)=(-s) \int_{0}^{\infty} \int_{0}^{\infty} e^{s t}(a(t-u)-a(t)) d t u^{-1} d \rho(u) \\
& =(-s) \int_{0}^{\infty} e^{s t}\left(\int_{0}^{\infty}(a(t-u)-a(t)) u^{-1} d \rho(u)\right) d t=(-s) \int_{0}^{\infty} e^{s t} b(t) d t=L b(s)
\end{aligned}
$$

(теорема Тонелли применима, так как из положительности меры $a$ следует, что ее функция распределения $a(t)$ не убывает, а потому $a(t-u)-a(t) \leq 0)$.

В частности, в силу теоремы 1 оператор $h(A)$ тоже ограничен.

С помощью интегрирования по частям легко проверить, что при $x \in D(A)$

$$
g(A) x=\int_{0}^{\infty} T(t)(-A x) a(t) d t
$$

Следовательно, при этих $x$ справедливы равенства

$$
\begin{aligned}
(T(u)-I) g(A) x & =\int_{0}^{\infty} T(u+t)(-A x) a(t) d t-\int_{0}^{\infty} T(t)(-A x) a(t) d t \\
& =\int_{0}^{\infty} T(t)(-A x)(a(t-u)-a(t)) d t .
\end{aligned}
$$

Поэтому при $x \in D(A)$ имеем по теореме Фубини для интеграла Бохнера

$$
\begin{gathered}
\psi(A) g(A) x \\
\left.=\int_{0}^{\infty}(T(u)-I)\right) g(A) x u^{-1} d \rho(u)=\int_{0}^{\infty}\left(\int_{0}^{\infty} T(t)(-A x)(a(t-u)-a(t)) d t\right) u^{-1} d \rho(u) \\
=\int_{0}^{\infty} T(t)(-A x)\left(\int_{0}^{\infty}(a(t-u)-a(t)) u^{-1} d \rho(u)\right) d t .
\end{gathered}
$$

Обоснуем применимость теоремы Фубини. Так как $\|T(t)(-A x)\| \leq M\|A x\| e^{-\sigma t}$, то это вытекает из равенства (см. (2.4))

$$
\int_{0}^{\infty}\left(\int_{0}^{\infty} e^{-\sigma t}(a(t-u)-a(t)) d t\right) u^{-1} d \rho(u)=\int_{0}^{\infty} \frac{\left(e^{-\sigma u}-1\right) g(-\sigma)}{\sigma} u^{-1} d \rho(u)=\frac{g(-\sigma)}{\sigma} \psi(-\sigma)<\infty
$$

обосновывающего абсолютную сходимость первого из повторных интегралов.

Следовательно, с учетом (2.5) имеем

$$
\psi(A) g(A) x=\int_{0}^{\infty} T(t)(-A x) b(t) d t=
$$




$$
=-\int_{0}^{\infty} b(t) d T(t) x=-\left.b(t) T(t) x\right|_{t=0} ^{\infty}+\int_{0}^{\infty} T(t) x d b(t)=h(A) x
$$

(мы воспользовались тем, что $b(t)=o\left(e^{\sigma t}\right), b(0)=0$ ), и при $x \in D(A)$ первое равенство доказано. Но, поскольку $D(A)$ есть существенная область для оператора $\psi(A)$ [17, теорема 1], оно верно при всех $x \in D(\psi(A))$.

Для доказательства второго равенства заметим, что операторы $T(u)$ и $g(A)$ коммутируют в том смысле, что при $x \in X$

$$
T(u) g(A) x=\int_{0}^{\infty} T(u+t) x d a(t)=\int_{0}^{\infty} T(t) T(u) x d a(t)=g(A) T(u) x,
$$

а потому при $x \in D(A)$

$$
\begin{aligned}
& h(A) x=\psi(A) g(A) x=\int_{0}^{\infty} g(A)(T(u)-I) x u^{-1} d \rho(u) \\
& \quad=g(A) \int_{0}^{\infty}(T(u)-I) x u^{-1} d \rho(u)=g(A) \psi(A) x .
\end{aligned}
$$

Как и выше, это равенство распространяется на все $x \in D(\psi(A))$.

Теорема доказана.

Следствие 9. Пусть $g \in L \mathcal{M}$ u полугруппа $T$ равномерно устойчива. Тогда $h(s):=$ $s g(s) \in L \mathcal{M}$ и при $x \in D(A)$ справедливы равенства $h(A) x=A g(A) x=g(A) A x$.

В самом деле, если в $(2.1)$ в качестве $\rho$ взять меру Дирака, то при $c_{0}=0$ получим $\psi(s)=s$. П р и м е р 2. Пусть $g(s)=(-s)^{-\alpha}, \psi(s)=-(-s)^{\beta}, 0<\beta<1, s<0, \alpha \geq \beta$. Как известно,

$$
(-s)^{-\alpha}=\frac{1}{\Gamma(\alpha+1)} \int_{0}^{\infty} e^{s t} d t^{\alpha}, \quad-(-s)^{\beta}=\frac{\beta}{\Gamma(1-\beta)} \int_{0}^{\infty}\left(e^{s u}-1\right) u^{-1-\beta} d u .
$$

Поскольку в этом случае $a(t)=c_{1} t^{\alpha}, u^{-1} d \rho(u)=c_{2} u^{-1-\beta} d u$, то при $t>0$

$$
\begin{aligned}
& b(t)=\int_{0}^{t} \frac{a(t-u)-a(t)}{u} d \rho(u)-a(t) \int_{t}^{\infty} u^{-1} d \rho(u) \\
& =c_{3} \int_{0}^{t} \frac{(t-u)^{\alpha}-t \alpha}{u} u^{-\beta} d u-c_{3} t^{\alpha} \int_{t}^{\infty} u^{-1-\beta} d u \\
& =c_{3} t^{\alpha-\beta}\left(\int_{0}^{1} \frac{(1-v)^{\alpha}-1}{v} \frac{d v}{v^{\beta}}-\frac{1}{\beta}\right)=c_{4} t^{\alpha-\beta} .
\end{aligned}
$$

Так как $\alpha \geq \beta$, то функция $b(t)$ имеет ограниченную вариацию на интервале $(0, R)$ при любом $R>0$ (но это не так при $\alpha<\beta$ ).

Если полугруппа $T$ равномерно устойчива, то все условия теоремы 4 выполнены. Тогда в силу этой теоремы для генератора $A$ полугруппы $T$ при всех $x \in D\left((-A)^{\beta}\right)$ справедливо равенство

$$
(-A)^{-\alpha}(-A)^{\beta} x=(-A)^{\beta-\alpha} x .
$$

В связи с идущим ниже следствием 10 отметим, что если $\psi \in \mathcal{T}$, то функция $-1 / \psi$ на $(-\infty, 0)$ абсолютно монотонна (см., например, [10, с. 27 , теорема $3.7(\mathrm{ii})])$, а потому по уже упоминавшейся теореме Бернштейна - Уиддера принадлежит $L \mathcal{M}$. 
Следствие 10. Пусть $\psi \in \mathcal{T}$ и полугруппа $T$ равномерно устойчива. Тогда оператор $\psi(A)$ обратим и $\psi(A)^{-1}=(1 / \psi)(A)$, где правая часть понимается в смысле определения 1.

Д о к а з а т е л ь с т в о. По теореме 1 оператор $(1 / \psi)(A)$ (в смысле определения 1 ) определен и ограничен на $X$. Так как $(1 / \psi)(s) \psi(s)=1$, то силу теоремы 3 при $x \in D(\psi(A))$ имеем

$$
(1 / \psi)(A) \psi(A) x=\psi(A)(1 / \psi)(A) x=x .
$$

Поскольку оператор $\psi(A)(1 / \psi)(A)$ замкнут как произведение замкнутого и ограниченного операторов, последнее равенство верно при всех $x \in X$. Следовательно, оператор $\psi(A)$ биективен и $\psi(A)^{-1}=(1 / \psi)(A)$.

Следствие доказано.

Идущее ниже следствие полезно сравнить с формулой Ноллау [18, теорема 7] для резольвенты логарифма оператора (см. также [15, теорема 9.1 и §11, пример 2]).

Следствие 11. Пусть полугруппа Т равномерно устойчива. Тогда существует ограниченный обратный оператор

$$
(\log (I-A))^{-1} x=\int_{1}^{\infty} \frac{(t-A)^{-1} x d t}{\pi^{2}+\log ^{2}(t-1)}+\int_{0}^{\infty} T(t) x d t \quad(x \in X) .
$$

В частности, если генератор А ингективен, то

$$
(\log (I-A))^{-1} x=\int_{1}^{\infty} \frac{(t-A)^{-1} x d t}{\pi^{2}+\log ^{2}(t-1)}-A^{-1} x \quad(x \in \operatorname{Im}(A)) .
$$

Д о к аз а т ел ь с т в о. Применим следствие 10 к функции $\psi(s)=-\log (1-s) \in \mathcal{T}$. Известно, что для функции

$$
\nu(t,-1)=\int_{0}^{\infty} \frac{t^{\xi-1}}{\Gamma(\xi)} d \xi
$$

справедливо равенство $1 / \log (-s)=\nu \widehat{(t,-1})(s)$ при $s<0[19$, гл. 5, §5.7, (11)]. Отсюда вытекает, что $1 / \psi=\widehat{(-f)}$, где $f(t)=e^{-t} \nu(t,-1)>0$. По следствию 10 существует ограниченный обратный оператор

$$
(\log (I-A))^{-1} x=\int_{0}^{\infty} T(t) x e^{-t} \nu(t,-1) d t \quad(x \in X) .
$$

Так как $T$ равномерно устойчива, то $\sigma(A) \subset\{\operatorname{Re}(z) \leq 0\}$, а потому при $t>0$

$$
(t-A)^{-1} x=\int_{0}^{\infty} e^{-t s} T(s) x d s .
$$

Следовательно, по теореме Фубини для интеграла Бохнера

$$
\begin{gathered}
J_{1}:=\int_{1}^{\infty} \frac{(t-A)^{-1} x d t}{\pi^{2}+\log ^{2}(t-1)}=\int_{1}^{\infty}\left(\int_{0}^{\infty} e^{-t s} T(s) x d s\right) \frac{d t}{\pi^{2}+\log ^{2}(t-1)} \\
=\int_{0}^{\infty} T(s) x d s \int_{1}^{\infty} \frac{e^{-t s}}{\pi^{2}+\log ^{2}(t-1)} d t .
\end{gathered}
$$


Применимость теоремы Фубини следует из оценки $\|T(s)\| \leq M e^{-\sigma s}(\sigma>0)$ и сходимости повторного интеграла

$$
\int_{1}^{\infty}\left(\int_{0}^{\infty} e^{-t s} e^{-\sigma s} d s\right) \frac{1}{\pi^{2}+\log ^{2}(t-1)} d t=\int_{1}^{\infty} \frac{1}{(t+\sigma)\left(\pi^{2}+\log ^{2}(t-1)\right)} d t .
$$

Но в силу [20, 2.6.27, формула 6]

$$
J_{2}:=\int_{1}^{\infty} \frac{e^{-t s}}{\pi^{2}+\log ^{2}(t-1)} d t=e^{-s} \int_{0}^{\infty} \frac{e^{-r s}}{\pi^{2}+\log ^{2} r} d r=e^{-s} \nu^{\prime}(s)-1,
$$

где

$$
\nu(s)=\int_{0}^{\infty} \frac{s^{t}}{\Gamma(t+1)} d t
$$

Значит,

$$
J_{2}=e^{-s} \int_{0}^{\infty} \frac{t s^{t-1}}{\Gamma(t+1)} d t-1=e^{-s} \nu(s,-1)-1
$$

а потому

$$
J_{1}=\int_{0}^{\infty} T(s) x\left(e^{-s} \nu(s,-1)-1\right) d s=(\log (I-A))^{-1} x-\int_{0}^{\infty} T(s) x d s,
$$

что доказывает первое утверждение следствия.

Если $A$ инъективен и $x \in \operatorname{Im}(A)$, то в силу примера 1 отсюда получим второе утверждение следствия.

Следствие доказано.

3 а м е ч а н и е 1 . Если в следствии 11 считать $A$ отрицательным числом и положить $s=t-1, z=1-A$, то приходим к равенству

$$
\int_{0}^{\infty} \frac{d s}{(s+z)\left(\pi^{2}+\log ^{2} s\right)}=\frac{1}{\log z}+\frac{1}{1-z} \quad(z>1)
$$

дающему, в частности, значение преобразования Стилтьеса (относительно последнего, см., например, $\left[16\right.$, гл. 8] или $\left[21\right.$, гл. 5]) функции $1 /\left(\pi^{2}+\log ^{2} s\right)$.

Теорема 5. Пусть $h=L a \in L \mathcal{M}$, где $a-$ положительная мера, $\psi \in \mathcal{T}$. Тогда $h \circ \psi \in L \mathcal{M}$ и если полугруппа $T$ равномерно устойчива, то

$$
(h \circ \psi)(A)=h(\psi(A))
$$

причем этот оператор ограничен.

Д о к а з а т е л ь с т в о. Ясно, что

$$
(h \circ \psi)(s)=\int_{0}^{\infty} e^{u \psi(s)} d a(u) .
$$

Но, как отмечалось выше,

$$
g_{u}(s)=\int_{0}^{\infty} e^{s t} d \nu_{u}(t)(s<0, u \geq 0)
$$


для некоторой субвероятностной непрерывной положительной меры $\nu_{u}$ на $\mathbb{R}_{+}$, причем по нашему соглашению $\nu_{u}(0)=0$. Интегрируя по частям, выводим отсюда, что

$$
g_{u}(s)=-s \int_{0}^{\infty} e^{s t} \nu_{u}(t) d t .
$$

Следовательно, меняя порядок интегрирпования в силу теоремы Тонелли, получаем при всех $s<0$

$$
\begin{gathered}
(h \circ \psi)(s)=\int_{0}^{\infty}\left((-s) \int_{0}^{\infty} e^{s t} \nu_{u}(t) d t\right) d a(u)=-s \int_{0}^{\infty} e^{s t}\left(\int_{0}^{\infty} \nu_{u}(t) d a(u)\right) d t \\
=\int_{0}^{\infty} e^{s t} d_{t}\left(\int_{0}^{\infty} \nu_{u}(t) d a(u)\right)=L \alpha(s)
\end{gathered}
$$

где $\alpha(t):=\int_{0}^{\infty} \nu_{u}(t) d a(u)$ (мы снова воспользовались теоремой 2.3а из [16, гл. 2]).

Таким образом, $h \circ \psi \in L \mathcal{M}$ и при $x \in X$

$$
(h \circ \psi)(A) x:=\int_{0}^{\infty} T(t) x d \alpha(t) .
$$

Отсюда, прежде всего, следует граниченность оператора $(h \circ \psi)(A)$, так как

$$
\|(h \circ \psi)(A) x\| \leq \int_{0}^{\infty}\|T(t) x\| d \alpha(t) \leq M(L \alpha)(-\sigma)\|x\| .
$$

Как и в доказательстве теоремы 4 , найдутся такие постоянные $M>0$ и $\sigma>0$, что $\|T(t)\| \leq$ $M e^{-\sigma t}$ при всех $t \in \mathbb{R}_{+}$, а поскольку $L \alpha(s)$ существует при всех $s<0$, то $\alpha(t)=o\left(e^{\sigma t}\right)$. Пусть $x \in D(A)$. Интегрируя по частям (и учитывая, что $\alpha(0)=0$ ), получаем из $(2.6)$ и теоремы Фубини, что

$$
\begin{gathered}
(h \circ \psi)(A) x=\left.T(t) x \alpha(t)\right|_{0} ^{\infty}-\int_{0}^{\infty} T^{\prime}(t) x \alpha(t) d t=-\int_{0}^{\infty} T^{\prime}(t) x \alpha(t) d t \\
=-\int_{0}^{\infty}\left(\int_{0}^{\infty} T^{\prime}(t) x \nu_{u}(t) d t\right) d a(u) .
\end{gathered}
$$

Обоснуем здесь применимость теоремы Фубини для интеграла Бохнера. Так как $\left\|T^{\prime}(t) x\right\|=$ $\|T(t) A x\| \leq M\|A x\| e^{-\sigma t}$, то достаточно доказать сходимость повторного интеграла

$$
\int_{0}^{\infty}\left(\int_{0}^{\infty} e^{-\sigma t} \nu_{u}(t) d t\right) d a(u)
$$

который в силу теоремы Тонелли равен

$$
\int_{0}^{\infty}\left(\int_{0}^{\infty} \nu_{u}(t) d a(u)\right) e^{-\sigma t} d t=L \alpha(-\sigma)
$$


Поскольку

$$
-\int_{0}^{\infty} T^{\prime}(t) x \nu_{u}(t) d t=-\left.T(t) x \nu_{u}(t)\right|_{0} ^{\infty}+\int_{0}^{\infty} T(t) x d \nu_{u}(t)=g_{u}(A) x
$$

то из $(2.7)$ вытекает, что $(h \circ \psi)(A) x=h(\psi(A)) x$ при $x \in D(A)$. По непрерывности это равенство верно при всех $x \in X$. В самом деле, ограниченность оператора, стоящего в левой части этого равенства, была доказана выше. Ограниченность же оператора, стоящего в правой части, будет следовать из теоремы 1 , если мы докажем, что оператор $\psi(A)$ есть генератор равномерно устойчивой полугруппы. Но это в самом деле так, поскольку, как отмечалось выше, этот оператор порождает $C_{0}$-полугруппу $g_{t}(A)=\int_{0}^{\infty} T(u) d \nu_{t}(u)$, где $g_{t}(s):=e^{t \psi(s)}=L \nu_{t}(s)$, а потому

$$
\left\|g_{t}(A)\right\| \leq \int_{0}^{\infty} M e^{-\sigma u} d \nu_{t}(u)=M e^{t \psi(-\sigma)} \rightarrow 0 \text { при } t \rightarrow+\infty
$$

в силу неравенства $\psi(-\sigma)<0$.

Теорема доказана.

П р и м е р 3. При $h(t)=(-t)^{-\alpha}, \psi(s)=-(-s)^{\beta}, \alpha>0, \beta \in(0,1)$, из предыдущей теоремы следует, что, если полугруппа $T$ равномерно устойчива, то

$$
\left((-A)^{\beta}\right)^{-\alpha}=(-A)^{-\beta \alpha} .
$$

\section{Заключение}

Таким образом, теоремы 3-5 устанавливают связь между классическими исчислениями Хилле - Филлипса и Бохнера - Филлипса. Отметим, что две последние теоремы доказаны для генераторов равномерно устойчивых полугрупп, что позволило получить в этом случае и правило обращения операторов в исчислении Бохнера-Филлипса (следствие 10). Представляло бы интерес обобщение теорем 4 и 5 на случай генераторов произвольных ограниченных (и сильно непрерывных) полугрупп операторов. Представляло бы интерес также ослабление условий теоремы 2 и получение условий замыкаемости операторов, возникающих в исчислении Хилле-Филлипса в смысле определения 1 данной работы.

Автор благодарит рецензента за замечания, способствовавшие улучшению изложения.

\section{СПИСОК ЛИТЕРАТУРЫ}

1. Хилле Э., Филлипс Р. Функциональный анализ и полугруппы. М. : ИЛ, 1962. 829 с.

2. Baeumer B., Haase M., Kovacs M. Unbounded functional calculus for bounded groups with applications // J. Evol. Equ. 2009. Vol. 9, no 1. P. 171-195.

3. Martinez-Carracedo C., Santz-Alix M. The theory of fractional powers of operators. Amsterdam: North Holland, 2001. 378 p.

4. Коркина Л.Ф., Рекант М.А. Свойства отображений скалярных функций в операторные линейного замкнутого оператора // Тр. Ин-та математики и механики УрО РАН. 2015. Т. 21, № 1. C. $153-165$.

5. Коркина Л.Ф., Рекант М.А. Некоторые классы функций линейного замкнутого оператора // Тр. Ин-та математики и механики УрО РАН. 2011. Т. 17, № 3. С. 186-200.

6. Миротин А.P. Об одном функциональном исчислении замкнутых операторов в банаховом пространстве. III. Некоторые вопросы теории возмущений // Изв. вузов. Математика. 2017. № 12. C. $24-34$.

7. Batty C.J.K., Gomilko A., Tomilov Yu. Product formulas in functional calculi for sectorial operators // Math. Z. 2015. Vol. 279. P. 479-507.

8. Batty C.J.K., Gomilko A., Tomilov Yu. Resolvent representations for functions of sectorial operators // Advances in Mathematics. 2017. Vol. 308. P. 896-940. 
9. Миротин А.P. О $\mathcal{T}$-исчислении генераторов $C_{0}$-полугрупп // Сибирский мат. журн. 1998. Т. 39, № 3. C. $571-583$.

10. Shilling R. L., Song R., Z. Vondrachek Z. Bernstein functions. Theory and applications. 2nd ed. Berlin; Boston: de Gruyter, 2012. 425 p.

11. Gomilko A., Tomilov Yu. On subordination of holomorphic semigroups // Advances in Mathematics. 2015. Vol. 283. P. 155-194.

12. Лопушанский О.В., Шарин С.В. Обобщенное функциональное исчисление типа ХиллеФиллипса для многопараметрических полугрупп // Сиб. мат. журн. 2014. Т. 55, № 1. С. 131-146.

13. Mirotin A.R. On the connections of Hille-Phillips functional calculus with Bochner-Phillips functional calculus [e-resource]. 2019. 14 p. URL: https://arxiv.org/pdf/1912.12423.pdf .

14. Като Т. Теория возмущений линейных операторов. Москва: Мир, 1972.740 с.

15. Миротин А.P. Многомерное $\mathcal{T}$-исчисление генераторов $C_{0}$-полугрупп // Алгебра и анализ. 1999. T. 11, № 2. C. 142-170.

16. Widder D.V. The Laplase transform. Prinston, 1946. 412 p.

17. Миротин A.P. Об отображении совместного спектра набора генераторов полугрупп // Функциональный анализ и его приложения. 2012. Т. 46, № 3. С. 62-70.

18. Nollau V. Über den Logarithmus abgeschlossener Operatoren in Banachschen Räumen // Acta Sci. Math. 1969. Vol. 30. P. 161-174.

19. Бейтмен Г., Эрдейи А. Таблицы интегральных преобразований. Т. 1. Преобразования Фурье, Лапласа, Меллина. Москва: Наука, 1969. 344 р.

20. Прудников А.П., Брычков Ю.А., Маричев О.И. Интегралы и ряды. Москва: Наука, 1981. $800 \mathrm{c.}$

21. Брычков Ю.А., Прудников А.П. Интегральные преобразования обобщенных функций. Москва: Наука, 1977. 288 с.

Миротин Адольф Рувимович

д-р физ.-мат. наук, профессор

зав. кафедрой

Гомельский государственный университет им. Ф. Скорины

г. Гомель

e-mail: amirotin@yandex.ru

\section{REFERENCES}

1. Hille E., Phillips R.S. Functional analysis and semi-groups. Colloquium Publications, vol. 31, Providence: American Mathematical Society, 1957, 808 p. ISBN: 0821874640. Translated to Russian under the title Funktsional'nyi analiz i polugruppy. Moscow: Inostr. Lit. Publ., 1962, 829 p.

2. Baeumer B., Haase M., Kovacs M. Unbounded functional calculus for bounded groups with applications. J. Evol. Equ., 2009, vol. 9, no. 1, pp. 171-195. doi: 10.1007/s00028-009-0012-z .

3. Martinez-Carracedo C., Santz-Alix M. The theory of fractional powers of operators. Amsterdam: North Holland, 2001, 378 p. doi: 10.1016/s0304-0208(00)x8025-5.

4. Korkina L.F., Rekant M.A. Properties of mappings of scalar functions to operator functions of a linear closed operator. Trudy Inst. Mat. i Mekh. UrO RAN, 2015, vol. 21, no. 1, pp. 153-165 (in Russian).

5. Korkina L.F., Rekant M.A. Some classes of functions of a linear closed operator. Proc. Steklov Inst. Math. (Suppl.), 2012, vol. 277, suppl. 1, pp. 121-135. doi: 10.1134/S0081543812050124.

6. Mirotin A.R. A functional calculus of closed operators on Banach space. III. Certain topics of perturbation theory. Russ Math., 2017, vol. 61, no. 12, pp. 19-28. doi: 10.3103/S1066369X17120039.

7. Batty C.J.K., Gomilko A., Tomilov Yu. Product formulas in functional calculi for sectorial operators. Math. Z., 2015, vol. 279, no. 1, pp. 479-507. doi: 10.1007/s00209-014-1378-3.

8. Batty C.J.K., Gomilko A., Tomilov Yu. Resolvent representations for functions of sectorial operators. Advances in Mathematics, 2017, vol. 308, pp. 896-940. doi: 10.1016/j.aim.2016.12.009. 
9. Mirotin A.R. On the $\mathcal{T}$-calculus of generators for $C_{0}$-semigroups. Sib. Math. J., 1998, vol. 39, no. 3, pp. 493-503. doi: 10.1007/BF02673905 .

10. Shilling R.L., Song R., Vondrachek Z. Bernstein Functions. Theory and Applications. 2nd ed. Berlin; Boston: de Gruyter, 2012, 425 p. doi: 10.1515/9783110269338.

11. Gomilko A., Tomilov Yu. On subordination of holomorphic semigroups. Advances in Mathematics, 2015, vol. 283, pp. 155-194. doi: 10.1016/j.aim.2015.05.016.

12. Lopushansky O.V., Sharyn S.V. Generalized Hille-Phillips type functional calculus for multiparameter semigroups. Siberian Math. J., 2014, vol. 55, no. 1, pp. 105-117. doi: 10.1134/S0037446614010133.

13. Mirotin A.R. On the connections of Hille-Phillips functional calculus with Bochner-Phillips functional calculus [e-resource], 2019, 14 p. Available at https://arxiv.org/pdf/1912.12423.pdf (in Russian).

14. Kato T. Perturbation theory for linear operators. Berlin: Springer-Verlag, 1995, 619 p. doi: 10.1007/978-3642-66282-9 . Translated to Russian under the title Teoriya vozmushchenii lineinykh operatorov. Moscow: Mir, 1972, $740 \mathrm{p}$.

15. Mirotin A.R. The multidimensional $\mathcal{T}$-calculus of generators of $C_{0}$-semigroups. St. Petersburg Math. J., 2000, vol. 11, no. 2, pp. 315-335.

16. Widder D.V. The Laplace transform. Princeton: Princeton University Press, 1946, 412 p.

17. Mirotin A.R. A Joint spectral mapping theorem for sets of semigroup generators. Funct. Anal. Appl., 2012, vol. 46, no. 3, pp. 210-217. doi: 10.4213/faa3076.

18. Nollau V. Über den Logarithmus abgeschlossener Operatoren in Banachschen Räumen. Acta Sci. Math., 1969, vol. 30, no. 3-4, pp. 161-174.

19. Bateman H., Erdelyi A. Tables of integral transforms. Vol. 1. N Y: McGraw-Hill, 1954, 391 p. ISBN: 0070195498. Translated to Russian under the title Tablitsy integral'nykh preobrazovanii. T. 1. Preobrazovaniya Fur'e, Laplasa, Mellina, Moscow: Nauka Publ., 1969, 344 p.

20. Prudnikov A.P., Brychkov Yu.A., Marichev O.I. Integrals and series. Vol. 1. Elementary functions. N Y etc.: Gordon and Breach, 1988, 798 p. ISBN: 9782881240898. Original Russian text published in Prudnikov A.P., Brychkov Yu.A., Marichev O.I. Integraly i ryady, Moscow: Nauka Publ., 1981, 800 p.

21. Brychkov Yu.A., Prudnikov A.P. Integral transforms of generalized functions. N Y; London: Gordon \& Breach, 1989, 342 p. ISBN: 2-88124-705-9 . Original Russian text published in Brychkov Yu.A., Prudnikov A.P. Integral'nye preobrazovaniya obobshchennykh funktsii, Moscow: Nauka Publ., 1977, 288 p.

Received May 30, 2020

Revised June 23, 2020

Accepted July 20, 2020

Adolf Ruvimovich Mirotin, Dr. Phys.-Math. Sci., Prof., Francisk Skorina Gomel State University, Gomel, 246019 Belarus, e-mail: amirotin@yandex.ru .

Cite this article as: A. R. Mirotin. On connections between the Bochner-Phillips and Hille-Phillips functional calculi, Trudy Instituta Matematiki $i$ Mekhaniki URO RAN, 2020, vol. 26, no. 3, pp. 118-132. 